\title{
Exercise-Based Cardiac Rehabilitation with and without Neuromuscular Electrical Stimulation and its Effect on Exercise Tolerance and Life Quality of Persons with Chronic Heart Failure
}

\author{
$b y$ \\ Cezary Kucio ${ }^{1,2}$, Petr Stastny³, Bożena Leszczyńska-Bolewska , \\ Małgorzata Engelmann ${ }^{1}$, Ewa Kucio ${ }^{1,5}$, Petr Uhlir ${ }^{6}$, Magdalena Stania ${ }^{1}$, \\ Anna Polak ${ }^{1,7}$
}

The study compares the effect of an exercise-based cardiac rehabilitation program with a program combining physical exercise and lower extremity neuromuscular electrical stimulation (NMES) on the recovery of patients with chronic heart failure (CHF) with NYHA II-III symptoms. Seventy two patients with stable CHF were randomly distributed to four groups that received exercise-based cardiac rehabilitation and pharmacological treatment. Groups I and II were additionally administered NMES (35 Hz and $10 \mathrm{~Hz}$, respectively) and in Group III sham NMES was applied. Group IV (controls) received solely pharmacological and exercise treatment. Exercise tolerance and quality of life were assessed in patients pre-treatment and at week 3. Three weeks of rehabilitation induced significant increases ( $p$ $<0.05)$ in the distance covered in the 6-minute walk test, the metabolic equivalent (MET), the duration of the treadmill exercise stress test, the left ventricle ejection fraction (LVEF) and improved quality of life in all groups, but betweengroup differences were not significant $(p>0.05)$. In none of the groups were the left ventricle end-systolic and enddiastolic diameters ( $\mathrm{mm}$ ) measured at week 3 significantly different from their baseline values $(p>0.05)$. Exercise-based cardiac rehabilitation contributed to higher exercise tolerance, LVEF and quality of life of CHF patients (NYHA II-III), contrary to cardiac rehabilitation combined with lower extremity NMES ( $35 \mathrm{~Hz}$ and $10 \mathrm{~Hz}$ ) that failed to induce such improvements. More research is necessary to assess the therapeutic efficacy of NMES applied to CHF patients with NYHA IV symptoms.

Key words: physical activity, cardiac rehabilitation, chronic heart failure, neuromuscular electrical stimulation, exercise tolerance, quality of life.

\section{Introduction}

Studies show that chronic heart failure $(\mathrm{CHF})$ is a major health concern worldwide.

Its main symptoms are dyspnea and fatigue limiting exercise tolerance, which in the early stage of the disease are only experienced during physical effort. The development of the

\footnotetext{
1 - Department of Physical Therapy, Academy of Physical Education in Katowice, Poland.

2 - Department of Internal Disease at the Multispecialty Hospital in Jaworzno, Poland.

3 - Laboratory of Physical Training Adaptation, Faculty of Physical Education and Sport, Charles University, Czech Republic.

4 - Silesian Rehabilitation Centre "Repty" in Tarnowskie Gory, Poland.

5 - Multispecialty Hospital in Siemianowice Slaskie, Poland.

6 - Department of Physiotherapy, Faculty of Physical Culture, Palacky University Olomouc, Czech Republic.

7 - Rehabilitation Center "Technomex", Gliwice, Poland.
} 
disease affects patients' daily lives, depriving them of self-reliance and making them believe that their lives have lower quality. CHF can also lead to disability and social isolation, as well as increasing the strain on the public healthcare system because CHF patients frequently require hospitalization. Its characteristics cause that CHF is both a medical and social problem (Ponikowski et al., 2016).

Studies show that exercise tolerance measured by peak oxygen uptake (VO2peak) is not related to cardiac function at peak exercise and that the causes of exercise dyspnea in CHF patients include skeletal muscle impairment (structural, metabolic or functional), decreased capillary density, peripheral arterial constriction, and reduced diastolic function of the heart during exercise. These observations have led to the creation of the "muscle hypothesis" for explaining chronic heart failure (Piepoli, 2013; Piepoli and Coats, 2007).

The recently published reports point out that the exercise-based cardiac rehabilitation of CHF patients is not only safe. but also offers many benefits, including higher exercise tolerance and $\mathrm{VO}_{2 \text { peak }}$ (Da Silva et al., 2002), decreased ergoreceptor reflexes, as well as increased baroreceptor reflexes (Ponikowski et al., 2001) and capillary density of skeletal muscles (Guazzi et al., 2005). It also improves the quality of life of CHF patients and reduces their need for assistance in activities of daily living (Flynn et al., 2009).

Research suggests that lower extremity neuromuscular electrical stimulation (NMES) can be an alternative to conventional physical training. NMES is reported to increase exercise tolerance, muscle mass and endurance, to improve muscular blood circulation, aerobic enzyme activity and vascular endothelium function, to decrease the concentration of proinflammatory cytokines, and to improve quality of life (Deley et al., 2008; Dobsak et al., 206; Karavidas et al., 2006, 2008; Soska et al., 2012). It is also safe for patients and carries a relatively low risk of dyspnea because it reduces exercise intensity. Additionally, NMES is easy to apply at home by a properly trained patient or caregiver. Its side effects include temporary muscle pain and a risk of skin being damaged by inappropriately attached electrodes.

This study was undertaken to expand the knowledge of cardiac rehabilitation applied to patients with $\mathrm{CHF}$ and to determine whether conventional exercise-based cardiac rehabilitation enhanced by lower extremity NMES $(35$ or $10 \mathrm{~Hz}$ ) can improve exercise tolerance, the levels of selected hemodynamic characteristics of the left ventricle (ejection fraction, end-diastolic and endsystolic diameters) and the quality of life of CHF patients.

\section{Methods}

\section{Participants}

The study participants were selected from patients with stable chronic heart failure (NYHA II-III symptoms) following stationary cardiac rehabilitation at the same rehabilitation center. Their eligibility to participate was assessed by their physician based on the inclusion and exclusion criteria presented in Table 1.

Patients' demographics were compiled from standard interviews, physical examinations, and medical records. Before the study, patients' body height, mass and body mass index (BMI) were determined. All patients performed a treadmill exercise stress test and were subjected to additional examinations including ECG, UCG (Vivid 4-GE Healthcare), and 24-hour cardiac monitoring (Oxford, Beng 2255A). To assess patients' blood cell morphology and blood concentration of glucose, total cholesterol, LDL, HDL, TG, creatinine and transaminases, standard blood tests were performed.

All patients who met the inclusion criteria were provided with written information about the purpose of the study and gave their consent to participate in it. Whether or not patients agreed to participate had no bearing on their further treatment.

Ethics approval for the study was granted by the Bioethics Commission at the Academy of Physical Education in Katowice, Poland (No. 5/2011).

\section{Design and Procedures}

The patients were randomly distributed to four groups. The Control Group was administered basic treatment consisting of exercise-based cardiac rehabilitation and pharmacological treatment. To the other three groups we additionally applied lower extremity NMES at a frequency of $35 \mathrm{~Hz}$ (the $35 \mathrm{~Hz} / \mathrm{NMES}$ Group), lower extremity NMES at $10 \mathrm{~Hz}$ (the 
10Hz/NMES Group) and sham lower extremity NMES (the sham/NMES Group).

Randomization. In allocating patients to groups the time and person to administer treatment were irrelevant factors. To carry out the allocation procedure, 4 sets of 20 slips of paper (numbered 1, 2, 3 and 4 for the respective study group) were placed in 80 envelops randomly drawn by the computer by an independent person who was unaware of the trial. Having been sealed, the envelopes were delivered to the main investigator who opened them consecutively in the presence of a physiotherapist to identify the group to which a patient was allocated.

\section{Intervention}

Cardiac rehabilitation. Patients in all groups received cardiac rehabilitation according to treatment models B, C and D as recommended by the European Society of Cardiology (Gianuzzi et al., 2001) and the European Association of Cardiovascular Prevention and Rehabilitation (Piepoli

et al., 2010). The models' protocols are presented in detail in Table 2.

Patients' performance in the treadmill exercise test pre-treatment was used to choose the relevant model of cardiac rehabilitation. Model C was found appropriate for 57 participants, of whom 15 received 35Hz/NMES, 11 10Hz/NMES, 16 sham/NMES and 15 patients were controls. Model D was applied to 15 patients, three of whom were administered $35 \mathrm{~Hz} / \mathrm{NMES}, 7$ patients received - 10Hz/NMES, 2 patients sham/NMES and 3 patients were controls. As the health of all patients covered by model D improved, model C was introduced instead. After 1 week, 4 patients rehabilitated according to model $\mathrm{C}$ were assigned model B on account of the progress they made.

To make sure that exercising was safe for patients, they were evaluated for cardiac failure severity before each session. The heart rate and blood pressure were assessed each morning and during exercise (together with fatigue measured with the Borg scale). Patients exercising on the cycle ergometer were ECG-monitored for signs of arrhythmia, atrioventricular conduction problems and/or significant changes in the ST segment.

Pharmacological treatment. Pharmacological treatment was optimized following the ESC guidelines (Ponikowski et al., 2016).

Neuromuscular electrical stimulation (NMES).
The NMES procedures were performed using the Firing device (Cosmogamma; Italy) that generated biphasic, symmetrical, rectangular pulses $(0.4 \mathrm{~ms})$ at a frequency of $35 \mathrm{~Hz}(35 \mathrm{~Hz} / \mathrm{NMES}$ Group) and $10 \mathrm{~Hz}(10 \mathrm{~Hz} / \mathrm{NMES}$ Group). Electrical stimulation was delivered to the quadriceps femoris and the triceps surae of both lower extremities via electrodes attached to the proximal and distal parts of the muscle bellies. Amperage was set individually for each patient at a level ensuring painless muscle contractions. A 2-s muscle contraction was followed by a 4-s rest period. First, both quadricepses were stimulated for 20 minutes, and then the triceps surae muscles for another 20 minutes under the supervision of a physiotherapist. The total time of a single NMES session was 40 minutes. Sessions were conducted 6 days a week over a period of 3 weeks (18 sessions).

Sham NMES. In the Sham/NMES Group, the electrodes were attached to patients' muscles in the same manner as in the other two NMES groups, but amperage was set to evoke tactile sensations instead of muscle contractions.

\section{Measures}

Exercise tolerance. To determine their exercise tolerance, patients performed a 6-minute walk test (6MWT) and an exercise stress test according to the modified Bruce protocol.

The 6MWT test was conducted in a $30-\mathrm{m}$ long, flat-surfaced hallway divided into 3-m segments and consisted of patients walking at a maximum comfortable speed for 6 minutes. The measure of their performance was the distance (in meters) they covered after 6 minutes (Hamilton et al., 2000).

The exercise stress test was performed on a treadmill in a standard manner (GE Medical System-Case Exercise Testing System) until patients scored a total of 15 points on the Borg's scale or exhibited symptoms requiring the test to be terminated. Statistical analysis was performed on the following exercise tolerance variables: the duration of a test and metabolic equivalent (MET).

The 6MWT and the treadmill test were carried out before rehabilitation commenced and at week 3 .

Echocardiographic screening. The echocardiographic screening procedure was performed using the transthoracic echocardiography method (VIVID 4, GE 
Healthcare). The hemodynamic variables assessed were the left ventricular ejection fraction (LVEF) and left ventricular end-systolic and end-diastolic diameters (LVESD and LVEDD, respectively). The procedure was carried out before the rehabilitation program and then repeated at week 3. Quality of life assessment (QL). Participants completed the Minnesota Living with Heart Failure Questionnaires (MLHFQ; license no. SKU Z94019A; Toy et al., 2000) measuring life quality in CHF patients at entry to the cardiac rehabilitation program and at week 4 . To ensure the reliability of their answers, explanations were provided whenever questions required clarification.

\section{Outcomes}

Primary outcome. The primary outcome of the study was patients' exercise tolerance at week 3 defined by their performance (distance) in the 6MWT, metabolic equivalent (MET) and the duration of the treadmill exercise stress test.

Secondary outcomes. The secondary outcomes of the study included: 1) cardiac function at week 3 defined by left ventricular function and hemodynamic variables (LVEF, LVESD and LVEDD); 2) patients' quality of life at week 4 determined from the MLHFQ scores.

\section{Statistical analysis}

Patients' characteristics were tested for normal distribution using the Shapiro-Wilk Wtest and the homogeneity of variance was verified by the Levene's test. Since distributions were normal and variances were homogenous, parametric tests were selected for statistical analysis. The homogeneity of the groups (in terms of patients' age, body height, body mass, and BMI) was tested pre-rehabilitation by means of one-way ANOVA where the independent variable represented the group to which a patient had been allocated. Mean results for the 6MWT and treadmill exercise test and the mean levels of MET, LVEF, LVESD, LVEDD and QL obtained before and after rehabilitation were tested by repeated measures ANOVA to make within- and between-group comparisons. The independent variables were the type of treatment and the time of measurement (before and after rehabilitation). The level of significance in all statistical analyses was set at $p \leq 0.05$. All analyses were performed using the Statistica software package 13.0 (StatSoft Polska Sp. z o.o.) by a person blinded to the type of rehabilitation.

\section{Results}

A group of 120 patients with CHF admitted for cardiac rehabilitation between the $1^{\text {st }}$ of December 2011 and the 31 $1^{\text {st }}$ of May 2013 was screened for eligibility for the study. Forty of them did not meet the inclusion criteria for reasons such as cardiac resynchronization therapy defibrillator implants (26), atrial ventricular pacemaker (10), and refusal to participate (4). The other 80 patients aged 36-79 with stable chronic heart failure (NYHA II-III) were randomly distributed to the following groups: $35 \mathrm{~Hz} / \mathrm{NMES}$ (20 persons), 10Hz/NMES (20 persons), Sham/NMES (19 persons) and Control (20 persons). One patient allocated to the Sham/NMES Group did not enter the rehabilitation program, having broken a leg. Of the remaining 79 participants, 7 (8.86\%) dropped out from the program; four (one in each group) because of exacerbation of heart failure symptoms and 3 (from the 35Hz/NMES, 10Hz/NMES and Control groups) voluntarily terminated their participation. Consequently, statistical analysis was performed on data obtained for four groups of 18 patients (72 in total). The flow of participants through the trial is shown in Figure 1.

Baseline characteristics. The study sample consisted of 72 CHF patients, 11 women $(15.28 \%)$ and 61 men $(84.72 \%)$ aged from 36 to 79 years. In 64 of them $(88.89 \%)$, the condition developed from coronary disease and in 8 (11.11\%) from valvular heart disease. The baseline assessments of CHF severity showed it to be moderate, corresponding to NYHA II (64 patients) and NYHA III (8 patients). The groups were similar at baseline $(p>0.05)$ in all characteristics but the BMI, which was significantly higher in the $35 \mathrm{~Hz} / \mathrm{NMES}$ and 10Hz/NMES Groups $(p<0.022)$ (Table 3).

\section{Primary outcomes}

\section{Exercise tolerance}

The 6MWT. The mean distance recorded at baseline for the 6MWT ranged from 375.3 (SD 72.32 ) to $416.7 \mathrm{~m}$ (SD 81.04) and did not significantly differentiate between the groups. After 3 weeks of rehabilitation, it increased to between 452.1 (SD 86.72) and 482.1m (SD 108.3). Significant progress was registered in all 4 groups $(p<0.0001)$, but repeated measures ANOVA showed that these improvements were not 
significantly different from each other $(p>0.05)$ (Table 4).

Cardiac stress test. The duration of the treadmill test $(\mathrm{t})$ ranged from 4.77 (SD 2.07) to 5.80 min (SD 2.25) and was not significantly different between the groups. Three weeks of rehabilitation significantly improved their performance on the test $(p<0.001)$, increasing its duration from 6.01 (SD 2.87) to $8.20 \mathrm{~min}$ (SD 2.57). As before, repeated measures ANOVA failed to reveal statistically significant between-group differences $(p>0.05)$ (Table 5). The mean metabolic equivalent (MET) obtained at baseline during the treadmill exercise test was in the range of 4.95 (SD 0.93 ) to 5.14 (SD 0.74$) \mathrm{ml} / \mathrm{kg} / \mathrm{min}$ and did not significantly differentiate between the groups. As a result of a three-week rehabilitation program its value significantly increased in all four groups ( $p$ $<0.05$ ) to between 5.17 (SD 0.78) and 5.47 (SD 1.10) $\mathrm{ml} / \mathrm{kg} / \mathrm{min}$. A repeated-measures ANOVA demonstrated that between-group differences were not statistically significant $(p>0.05)$ (Table 4).

\section{Secondary outcomes}

Echocardiographic test. The study groups were not significantly different at baseline in the left ventricular ejection fraction (LVEF), which ranged from 36.6 (SD 5.34) to $38.3 \%$ (SD 4.68). Three weeks of a rehabilitation program significantly increased LVEF in all groups $(p<$ $0.0001)$, its post intervention values ranging between 38.4 (SD 5.08) and 40.0\% (SD 4.95). Despite the change, the mean values of LVEF were not significantly different between the groups ( $p>0.05$; Table 5).

The left ventricular end-diastolic and endsystolic diameters (LVEDD and LVESD) measured at baseline and at week 3 were not significantly different from each other in any of the groups ( $p>0.05$; Table 5).

Quality of life. At baseline, patients' scores on the MLHFQ varied between 22.7 (SD 12.86) and 31.6 (SD 10.01) and were not significantly different between the groups (Table 6). The postrehabilitation scores were significantly lower in all groups ( $p<0.0001$ ), ranging between 13.5 (SD 8.16) and 20.8 (SD 12.49) points. The betweengroup differences were not statistically significant $(p>0.05 ;$ Table 6).

\section{Discussion}

From the review of recent studies presented by Banerjee (2010) it follows that lower extremity NMES can be an alternative to conventional exercise-based rehabilitation of $\mathrm{CHF}$ patients during exacerbation of the disease, particularly in patients with NYHA IV symptoms and patients before and after heart transplants. NMES is also reported to promote the rehabilitation of $\mathrm{CHF}$ patients with motor system disorders preventing them from taking conventional exercise and $\mathrm{CHF}$ patients with decreased left ventricular ejection fraction with NYHA II and III symptoms.

According to available reports, in patients with CHF NMES can improve peak oxygen uptake, anaerobic threshold, the duration of the exercise tolerance test, and performance on the 6MWT test (Deley et al., 2008; Dobsak et al., 2006; Karavidas et al., 2008, 2010; Soska et al., 2012). A series of NMES procedures has been found to improve muscle endurance to fatigue in these patients (Harris et al., 2003). In one study, the strength of stimulated muscles during isometric and isokinetic contractions increased from baseline by an average of 11-20\% (Dobsak et al., 2006). The changes are associated with higher aerobic enzyme activity increasing the oxidative capacity of the skeletal muscles (Dobsak et al., 2006; Harris et al., 2003; Karavidas et al., 2006, 2008; Nuhr et al., 2004). An examination of muscle biopsies led Nurh et al. (2004) to the conclusion that NMES increases the number of type I muscle fibers, thereby improving the muscle aerobic metabolism and endurance capacity.

NMES is also reported to increase blood circulation in the stimulated muscles (Dobsak et al., 2006), probably by improving the ratio of antiinflammatory cytokine IL-10 to pro-inflammatory cytokine TNF- $\alpha$ (Karavidas et al., 2006). Moreover, CHF patients treated with NMES present significantly lower levels of dissolved adhesion molecules, for instance soluble intercellular adhesion molecule-1 (sICAM-1) and soluble vascular adhesion molecule-1 (sVCAM-1), both of which are the "final product" of interaction between active monocytes and endothelial cells (Karavidas et al., 2006). 
Table 1

Inclusion and exclusion criteria for the study.
Inclusion criteria
Exclusion criteria

1. Documented history of chronic heart 1. Unstable coronary disease. failure. 2 . Severe aortic valve stenosis.

2. Left ventricular ejection fraction $<45 \%$ 3. Valvular impairment requiring surgical according to electrocardiography. intervention.

3. NYHA II-III.

4. Clinically stable CHF.

5. Consent to participate.

4. Complex ventricular arrhythmia.

5. Implanted cardiostimulator, ICD and CRT.

6. Uncontrolled hypertension.

7. Venous thromboembolism.

8. Exacerbation of chronic obstructive pulmonary disease.

9. Exacerbation of liver or kidney disease.

10. Lack of consent.

CHF - chronic heart failure; ICD - cardioverter defibrillator; $C R T$ - cardiac resynchronization therapy device

\section{Table 2}

Models of cardiac rehabilitation applied to the CHF patients.

\begin{tabular}{|c|c|c|c|}
\hline $\begin{array}{l}\text { Cardiac } \\
\text { rehabilitatio } \\
n \text { model }\end{array}$ & $\begin{array}{l}\text { MET level } \\
\text { during the } \\
\text { exercise stress } \\
\text { test }\end{array}$ & Rehabilitation protocol & Exercise intensity \\
\hline B & $>5 \mathrm{MET}$ & $\begin{array}{l}\text { - endurance or interval training } \\
\text { 3-5 times/week, } 45-60 \mathrm{~min} / \text { day, } \\
\text { - resistance training 2-3 times/week, } \\
\text { - general conditioning exercises } \\
5 \text { times/week. }\end{array}$ & $\begin{array}{l}50-60 \% \text { of heart rate } \\
\text { reserve }\end{array}$ \\
\hline $\mathrm{C}$ & 3-5 MET & $\begin{array}{l}\text { - endurance training or interval } \\
\text { training } \\
3-5 \text { times/week, } 45 \mathrm{~min} / \text { day, } \\
\text { - general conditioning exercise } \\
5 \text { times/week. }\end{array}$ & $\begin{array}{l}40-50 \% \text { of heart rate } \\
\text { reserve. }\end{array}$ \\
\hline $\mathrm{D}$ & $<3 \mathrm{MET}$ & $\begin{array}{l}\text { - individual exercise regimen, } \\
\text { - breathing exercises, } \\
\text { - walks. }\end{array}$ & $\begin{array}{l}\text { below } 20 \% \text { of heart rate } \\
\text { reserve. }\end{array}$ \\
\hline
\end{tabular}


Table 3

Baseline patient characteristics $(N=72)$.

$\begin{array}{ccccc}\text { Variable } & \text { 35Hz/NMES Group } & \text { 10Hz/NMES Group } & \text { Sham } & \text { Control Group } \\ (\mathrm{n}=18) & (\mathrm{n}=18) & (\mathrm{n}=18) & (\mathrm{n}=18)\end{array}$

Mean (SD)

\begin{tabular}{|c|c|c|c|c|}
\hline${ }^{1}$ Age (years) & $59.7(8.97)$ & $65.9(7.34)$ & $62.9(7.84)$ & $64.3(6,98)$ \\
\hline${ }^{1}$ Height $(\mathrm{m})$ & $1.71(0.072)$ & $1.69(0.077)$ & $1.68(0.061)$ & $1.69(0.072)$ \\
\hline${ }^{1}$ Body mass (kg) & $80.8(13.76)$ & $81.8(15.45)$ & 75.7 (11.92) & $70.2(12.08)$ \\
\hline${ }^{1} \mathrm{BMI}\left(\mathrm{kg} / \mathrm{m}^{2}\right)$ & $28.2(4.42)^{*}$ & $28.3(4.38)^{*}$ & $27.2(3.92)$ & $24.4(3.53)$ \\
\hline 26MWT (m) & $416.7(81.04)$ & $375.0(72.32)$ & $402.9(92.82)$ & $395.6(63.73)$ \\
\hline $2 \mathrm{t}(\mathrm{min})$ & $5.65(2.20)$ & $5.78(2.44)$ & $5.80(2.25)$ & $4.77(2.07)$ \\
\hline${ }^{2} \mathrm{MET}(\mathrm{ml} / \mathrm{kg} / \mathrm{min})$ & $5.00(1.05)$ & $5.09(1.28)$ & $4.95(0.93)$ & $5.14(0.74)$ \\
\hline${ }^{2} \mathrm{LVEF}(\%)$ & $38.3(4.68)$ & $37.3(4.00)$ & $36.6(5.34)$ & $37.7(3.86)$ \\
\hline${ }^{2} \operatorname{LVEDD}(\mathrm{mm})$ & $55.3(6.13)$ & $57.1(6.23)$ & $57.6(5.03)$ & $57.7(6.12)$ \\
\hline${ }^{2} \operatorname{LVESD}(\mathrm{mm})$ & $40.5(8.49)$ & $43.5(7.76)$ & $40.6(7.19)$ & $44.3(8.11)$ \\
\hline${ }^{2} \mathrm{MLHFQ}$ (points) & $24.4(12.43)$ & $22.7(12.86)$ & $29.1(14.20)$ & $31.6(10.01)$ \\
\hline
\end{tabular}

NMES - neuromuscular electrical stimulation; BMI - Body Mass Index; 6MWT - 6-minute walk test;

$t$ - the duration of the exercise stress test; MET - metabolic equivalent;

LVEF - left ventricular ejection fraction, LVEDD - left ventricular end-diastolic diameter;

LVESD - left ventricular end-systolic diameter;

MLHFQ - Minnesota Living with Heart Failure Questionnaire (a quality of life test);

${ }^{1}$ One-factor ANOVA;

${ }^{*} B M I$ statistically significant different with Sham/NMES

and Control Groups $(p<0.022)$

${ }^{2}$ Repeated measures ANOVA 
Table 4

Groups' results on the 6MWT test and exercise stress test performed according to the modified Bruce protocol before and after rehabilitation $(N=72)$.

\section{Variable}

Mean (SD)

\begin{tabular}{|c|c|c|c|c|c|c|}
\hline \multirow[t]{2}{*}{$\begin{array}{l}{ }^{16 \mathrm{MWT}} \\
(\mathrm{m})\end{array}$} & Baseline & 416.7 (81.04) & $375.0(72.32)$ & 402.9 (92.82) & $395.6(63.73)$ & $p>0.05$ \\
\hline & At week 3 & 481.9 (85.77) & $452.1(86.72)$ & $482.1(108.3)$ & $465.0(70.27)$ & \multirow[t]{2}{*}{$p>0.05$} \\
\hline & ${ }^{*} \mathrm{p}$ & $p<0.001$ & $p<0.001$ & $p<0.001$ & $p<0.001$ & \\
\hline \multirow{3}{*}{$\begin{array}{l}1 \mathrm{t} 2 \\
(\mathrm{~min})\end{array}$} & Baseline & $5.65(2.20)$ & $5.78(2.44)$ & $5.80(2.25)$ & 4.77 (2.07) & $p>0.05$ \\
\hline & At week 3 & $8.20(2.57)$ & $7.63(2.60)$ & $8.03(2.25)$ & $6.01(2.87)$ & \multirow[t]{2}{*}{$p>0.05$} \\
\hline & ${ }^{*} \mathrm{p}$ & $p<0.001$ & $p<0.001$ & $p<0.001$ & $p<0.001$ & \\
\hline \multirow{3}{*}{$\begin{array}{l}{ }^{1} \mathrm{MET} \\
(\mathrm{ml} / \mathrm{kg} / \mathrm{min})\end{array}$} & Baseline & $5.00(1.05)$ & $5.09(1.28)$ & $4.95(0.93)$ & $5.14(0.74)$ & $p>0.05$ \\
\hline & At week 3 & $5.32(1.48)$ & $5.17(0.78)$ & $5.47(1.10)$ & $5.17(0.81)$ & \multirow[t]{2}{*}{$p>0.05$} \\
\hline & ${ }^{*} \mathrm{p}$ & $p<0.05$ & $p<0.05$ & $p<0.05$ & $p<0.05$ & \\
\hline \multicolumn{7}{|c|}{$\begin{array}{c}{ }^{*} p-\text { within group level of significance; } 6 M W T-6 \text {-minute walk test; } \\
t-\text { duration of the exercise stress test; } \\
\text { TR-metabolic equivalent; NMES - neuromuscular electrical stimulation. } \\
\text { 'Repeated measures ANOVA. }\end{array}$} \\
\hline
\end{tabular}


Table 5

Groups' results of echocardiographic examination conducted before and after rehabilitation $(N=72)$. Variable

$\begin{array}{ccccc}35 \mathrm{~Hz} / \mathrm{NMES} & \text { 10Hz/NMES } & \text { Sham/NMES } & \text { Control } & \text { Between- } \\ \begin{array}{c}\text { Group } \\ (\mathrm{n}=18)\end{array} & \text { Group } & \text { Group } & \text { Group } & \text { group } \\ & (\mathrm{n}=18) & (\mathrm{n}=18) & (\mathrm{n}=18) & \begin{array}{c}\text { significance } \\ \text { level }\end{array}\end{array}$

Mean (SD)

\begin{tabular}{|c|c|c|c|c|c|c|}
\hline \multirow{3}{*}{$\begin{array}{l}{ }^{1} \mathrm{LVEF} \\
(\%)\end{array}$} & Baseline & $38.3(4.68)$ & $37.3(4.00)$ & $36.6(5.34)$ & 37.7 (3.86) & $p>0.05$ \\
\hline & At week 3 & $40.0(4.95)$ & $39.3(4.16)$ & $38.4(5.08)$ & $39.2(5.24)$ & $p>0.05$ \\
\hline & ${ }^{*} p$ & $p<0.0001$ & $p<0.0001$ & $p<0.0001$ & $p<0.0001$ & \\
\hline \multirow{3}{*}{$\begin{array}{l}{ }^{1} \text { LVEDD } \\
(\mathrm{mm})\end{array}$} & Baseline & $55.3(6.13)$ & $57.1(6.23)$ & $57.6(5.03)$ & $57.7(6.12)$ & $p>0.05$ \\
\hline & At week 3 & $54.5(6.22)$ & $56.8(6.25)$ & $56.6(4.27)$ & $57.5(6.73)$ & $p>0.05$ \\
\hline & ${ }^{*} p$ & $p>0.05$ & $p>0.05$ & $p>0.05$ & $p>0.05$ & \\
\hline \multirow{3}{*}{$\begin{array}{l}{ }^{1} \mathrm{LVESD} \\
(\mathrm{mm})\end{array}$} & Baseline & 40.5 (8.49) & $43.5(7.76)$ & $40.6(7.19)$ & $44.3(8.11)$ & $p>0.05$ \\
\hline & At week 3 & $38.9(8.37)$ & $42.1(7.35)$ & 40.7 (6.85) & $43.9(7.66)$ & $p>0.05$ \\
\hline & ${ }^{*} p$ & $p>0.05$ & $p>0.05$ & $p>0.05$ & $p>0.05$ & \\
\hline
\end{tabular}

${ }^{*} p$-within group level of significance; NMES - neuromuscular electrical stimulation; $L V E F$ - left ventricular ejection fraction, $L V E D D$ - left ventricular end-diastolic diameter; LVESD - left ventricular end-systolic diameter; ${ }^{1}$ Repeated measures ANOVA.

Table 6

Quality of life of patients with CHF pre- and post-rehabilitation $(N=72)$.

Variable

$\begin{array}{ccccc}\text { 35Hz/NMES } & 10 \mathrm{~Hz} / \mathrm{NMES} & \text { Sham/NMES } & \text { Control } & \text { Between-group } \\ \text { Group } & \text { Group } & \text { Group } & \text { Group } & \text { significance } \\ (\mathrm{n}=18) & (\mathrm{n}=18) & (\mathrm{n}=18) & (\mathrm{n}=18) & \text { level }\end{array}$

Mean (SD)

$\begin{array}{lllllll}\begin{array}{l}\text { MLHFQ } \\ \text { (Points) }\end{array} & \text { Baseline } & 24.4(12.43) & 22.7(12.86) & 29.1(14.20) & 31.6(10.01) & p>0.05 \\ & \text { At week 3 } & 15.3(9.95) & 13.5(8.16) & 20.8(12.49) & 19.4(8.53) & p>0.05 \\ & * p & p<\mathbf{0 . 0 0 0 1} & p<\mathbf{0 . 0 0 0 1} & p<\mathbf{0 . 0 0 0 1} & p<0.0001 & \end{array}$

${ }^{*} p$ - within group level of significance; NMES - neuromuscular electrical stimulation; MLHFQ - Minnesota Living with Heart Failure Questionnaire. Repeated measures ANOVA. 


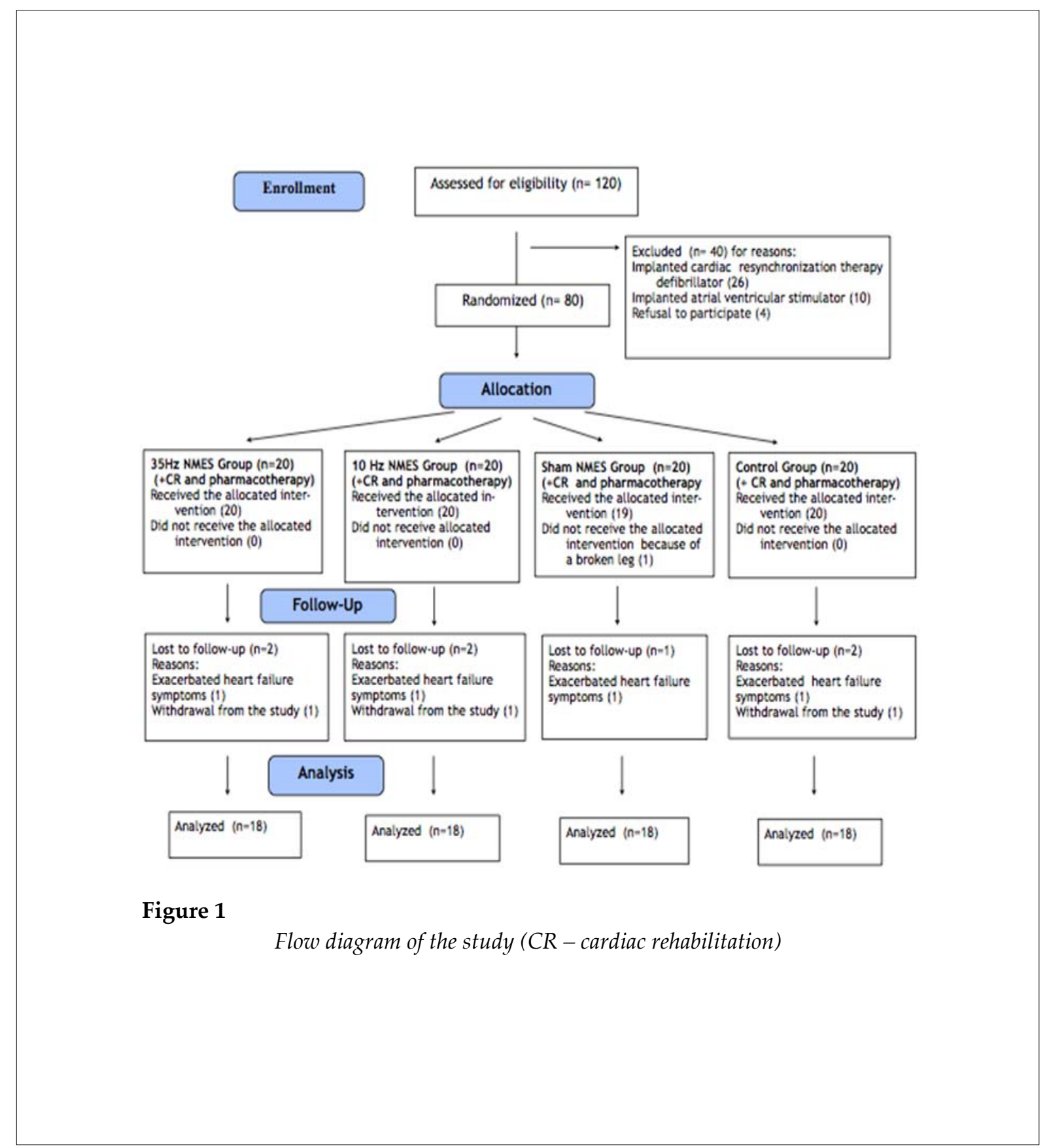

According to Karavidas et al. (2008), NMES improves vascular endothelium function and increases exercise capacity in CHF patients, thus breaking the vicious circle between abnormal peripheral inflammatory responses, abnormalities of the nitric oxide metabolism and vascular muscle dysfunction.

In two meta-analyses (Sbruzzi et al., 2010; Smart et al., 2012), lower extremity NMES, cycle ergometer exercise (Sbruzzi et al., 2010) and exercise combined with sham electrical stimulation (Smart et al., 2012) were compared for their effect on selected exercise tolerance variables in $\mathrm{CHF}$ patients. Cycle ergometer exercise increased their peak oxygen uptake (VO2peak), performance on the 6MWT and strength of thigh muscle contractions significantly more than lower extremity NMES, which, in turn, proved significantly more effective in improving these endurance variables than sham electrical stimulation (Smart et al., 2012).

This study was undertaken to assess and 
compare the efficacy of exercise-based cardiac rehabilitation enhanced by lower extremity NMES $(35 \mathrm{~Hz}$ and $10 \mathrm{~Hz}$ ), exercise-based cardiac rehabilitation and sham NMES in CHF patients. A 3-week rehabilitation program improved patients' performance on the 6MWT, increased the duration of the treadmill test and MET levels recorded during a treadmill test in all groups, but the levels of exercise tolerance variables were not significantly different between the groups. The influence of NMES on patients' exercise tolerance was not found to vary depending on its frequency $(35 \mathrm{~Hz}$ and $10 \mathrm{~Hz}$ ). The study findings indicate that conventional exercise-based cardiac rehabilitation enhanced by NMES $(35 \mathrm{~Hz}$ or 10 $\mathrm{Hz}$ ) is not effective in improving exercise tolerance in CHF patients with NYHA II or III symptoms.

This conclusion is consistent with the report by Soska et al. (2012) who also applied physical exercise, NMES of $10 \mathrm{~Hz}$ alone and conventional cardiac rehabilitation to $\mathrm{CHF}$ patients, and did not find statistically significant differences in their exercise tolerance.

Physical exercise is reported to improve function of the heart muscle. In Hambrecht et al.'s study (2000), physical exercise increased the resting heart rate and cardiac output in CHF patients, which the authors attributed to reduced end-systolic volume of the left ventricle and peripheral arterial resistance. According to another study, physical exercise in CHF patients counteracts the remodeling of the left ventricle, probably due to its mitigating effect on neurohormonal disorders (Giannuzzi et al., 2003).

In this study, the inclusion of NMES (35 $\mathrm{Hz}$ and $10 \mathrm{~Hz}$ ) in a conventional exercise-based cardiac rehabilitation program did not improve hemodynamic indicators (left ventricular ejection fraction (LVEF), end-diastolic diameter (LVEDD), and end-systolic diameter (LVESD)) in patients with CHF.

The most common causes of hospitalization and the inferior quality of life of CHF patients include exercise intolerance, fatigue, exercise dyspnea and resting dyspnea. They also report to be overwhelmed by the disease and to have little control over their lives. The stress they experience affects their emotional, physical and mental wellbeing.

In assessing individuals' quality of life, their frame of mind, physical and mental health, and social functioning need to be considered. The Minnesota Living with Heart Failure Questionnaire (MLHFQ) has been developed specifically to measure all these dimensions in CHF patients. The MLHFQ is a self-assessment tool containing 21 questions that allow CHF patients to make a structured and comprehensive overview of how heart failure and the treatment they receive affect their daily lives.

The quality of life and physical capacity of CHF patients can be improved by moderate physical exercise (Flynn et al., 2009; Van den BergEmons et al., 2005), as well as by NMES applied to the skeletal muscles of the lower extremities that increases their strength (Karavidas et al., 2008; Soska et al., 2012). The 'trained' patients perceive themselves as physically and mentally stronger, which gives them the feeling of being more independent in carrying out daily activities. However, for the benefits of a short-term exercise program to be sustainable, a lasting change in the lifestyle is necessary.

The similarity of groups' scores on the MLHFQ performed at week 3 implies that including NMES $(35 \mathrm{~Hz}$ and $10 \mathrm{~Hz})$ in exercisebased cardiac rehabilitation does not make it more efficacious in improving the quality of life of CHF patients. This observation is consistent with the findings of the study by Soska et al. (2012), who also used conventional cardiac rehabilitation in conjunction with NMES $(10 \mathrm{~Hz})$.

Limitations of the study. One limitation of the study is the short period of observation related to the National Health Fund limiting its grant to 3 weeks of stationary cardiac rehabilitation. It was long enough for the rehabilitation program to significantly improve CHF patients' exercise tolerance, left ventricular ejection fraction and life quality, but apparently too short to demonstrate whether conventional exercise-based cardiac rehabilitation enhanced by lower extremity NMES can improve the levels of hemodynamic indicators such as LVEDD and LVESD.

Because of ethical considerations, all patients in this study received conventional cardiac rehabilitation. This prevented us from evaluating the effect of lower extremity NMES alone on their health and wellbeing. More research is therefore necessary, because NMES 
seems to perform well as a therapy for cardiac patients who cannot exercise.

\section{Conclusions}

The study demonstrated that exercisebased cardiac rehabilitation combined with pharmacological treatment increased exercise tolerance, left ventricular ejection fraction and the quality of life of CHF patients with NYHA II-III symptoms.
Exercise-based cardiac rehabilitation enhanced by lower-extremity NMES (35 and 10 $\mathrm{Hz}$ ) failed to improve exercise tolerance, the quality of life and cardiac hemodynamic function in the studied CHF patients.

More research is necessary to determine the efficacy of NMES applied to patients with advanced CHF (NYHA IV).

\section{Acknowledgements}

Research activities were funded by the Academy of Physical Education in Katowice (Poland) and from grants: UNCE/HUM/032 and PRIMUS/17/MED/5.

\section{References}

Banerjee P, Caulfield B, Crowe L, Clark AL. Prolonged electrical muscle stimulation exercise improves strength, peak $\mathrm{VO}_{2}$, and exercise capacity in patients with stable chronic heart failure. J Card Fail, 2009; 15(4): 319-326

Da Silva MS, Bocchi EA, Guimaraes GV, Padovani CR, Gaiva MH, Pereira SF, Fontes RD. Benefits of exercise training in the treatment of heart failure. Study with a control group. Arq Bras Cardiol, 2002; 79: 357362

Deley G, Eicher JC, Verges B, Wolf JE, Casillas JM. Do low-frequency electrical myostimulation and aerobic training similarly improve performance in chronic heart failure patients with different exercise capacities? J Rehabil Med, 2008; 40: 219-224

Dobsak P, Navakova M, Fiser B, Siegelová J, Balcárková P, Spinarová L, Vítovec J, Minami N, Nagasaka M, Kohzuki M, Yambe T, Imachi K, Nitta S, Eicher JC, Wolf JE. Electrical stimulation of skeletal muscles. An alternative to aerobic exercise training in patients with chronic heart failure? Int Heart J, 2006; 47: 441-453

Dobsak P, Novakova M, Siegelova J, Fiser B, Vítovec J, Nagasaka M, Kohzuki M, Yambe T, Nitta S, Eicher JC, Wolf JE, Imachi K. Low-frequency electrical stimulation increases muscle strength and improves blood supply in patients with chronic heart failure. Circ J, 2006; 70: 75-82

Flynn KE, Pina IL, Whellan DJ, Lin L, Blumenthal JA, Ellis SJ, Fine LJ, Howlett JG, Keteyian SJ, Kitzman DW, Kraus WE, Miller NH, Schulman KA, Spertus JA, O'Connor CM, Weinfurt KP; HF-ACTION Investigators. Effects of exercise training on health status in patients with chronic heart failure. HFACTION randomized Controlled trial. JAMA, 2009; 302: 1451-1459

Giannuzzi P, Temporelli PL, Corra U, Tavazzi L; ELVD-CHF Study Group. Antiremodeling effect of longterm exercise training in patients with stable chronic heart failure. Results of the exercise in left ventricular dysfunction and chronic heart failure (ELVD-CHF) trial. Circulation, 2003; 108: 554-559

Gianuzzi P, Tavazzi L. Recommendation for exercise training in chronic heart failure patients. Working Group on Cardiac Rehabilitation \& Exercise Physiology and Working Group on Heart Failure of the European Society of Cardiology. Eur Heart J, 2001; 22: 115-135

Guazzi M, Reina G, Tumminello G, Guazzi MD. Exercise ventilation inefficiency and cardiovascular mortality in heart failure: the critical independent prognostic value of the arterial $\mathrm{CO}_{2}$ partial pressure. Eur Heart J, 2005; 26: 472-480

Hambrecht R, Gielen S, Linke A, Fiehn E, Yu J, Walther C, Schoene N, Schuler G. Effects of exercise training on left ventricular function and peripheral resistance in patients with chronic heart failure a randomized trial. JAMA, 2000; 283: 3095-3101 
Hamilton DM, Heannel RG. Validity and reliability of the 6-minute walk test in a cardiac rehabilitation. J Cardiopulm Rehabil, 2000; 20: 156-164

Harris S, LeMaitre JP, Mackenzie G, Fox KA, Denvir MA. A randomised study of home-based electrical stimulation of the legs and conventional bicycle exercise training for patients with chronic heart failure. Eur Heart J, 2003; 24: 871-878

Karavidas A, Parissis J, Arapi S, Farmakis D, Korres D, Nikolaou M, Fotiadis J, Potamitis N, Driva X, Paraskevaidis I, Matsakas E, Filippatos G, Kremastinos DT. Effects of functional electrical stimulation on quality of life and emotional stress in patients with chronic heart failure secondary to ischaemic or idiopathic dilated cardiomyopathy: a randomised, placebo-controlled trial. Eur J Heart Fail, 2008; 10: 709-713

Karavidas A, Parissis JT, Matzaraki V, Arapi S, Varounis C, Ikonomidis I, Grillias P, Paraskevaidis I, Pirgakis V, Filippatos G, Kremastinos DT. Functional electrical stimulation is more effective in severe symptomatic heart failure patients and improves their adherence to rehabilitation programs. J Card Fail, 2010; 16(3): 244-249

Karavidas A, Raisakis KG, Parissis JT, Tsekoura DK, Adamopoulos S, Korres DA, Farmakis D, Zacharoulis A, Fotiadis I, Matsakas E, Zacharoulis A. Functional electrical stimulation improves endothelial function and reduces peripheral immune responses in patients with chronic heart failure. Eur J Cardiovasc Prev Rehabil, 2006; 13(4): 592-597

Nuhr MJ, Pette D, Berger R, Quittan M, Crevenna R, Huelsman M, Wiesinger GF, Moser P, Fialka-Moser V, Pacher R. Beneficial effects of chronic low-frequency stimulation of thigh muscles in patients with advanced chronic heart failure. Eur Heart J, 2004; 25: 136-143

Piepoli MF, Coats AJS. Counterpoint: Increased metaboreceptor stimulation explains the exaggerated exercise pressor reflex seen in heart failure. J Appl Physiol, 2007; 102: 494-496

Piepoli MF, Corra U, Benzer W, Bjarnason-Wehrens B, Dendale P, Gaita D, McGee H, Mendes M, Niebauer J, Zwisler AD, Schmid JP. Cardiac Rehabilitation Section of the European Association of Cardiovascular Prevention and Rehabilitation. Secondary prevention through cardiac rehabilitation: from knowledge to implementation. A position paper from the Cardiac Rehabilitation Section of the European Association of Cardiovascular Prevention and Rehabilitation. Eur J Cardiovasc Prev Rehabil, 2010; 17(1): 7-8

Piepoli MF. Exercise training in chronic heart failure: mechanism and therapies. Neth Heart J, 2013; 21: 85-90

Ponikowski P, Voors AA, Anker SD, Bueno H, Cleland JGF, Coats AJS, Falk V, González-Juanatey JR, Harjola VP, Jankowska EA, Jessup M, Linde C, Nihoyannopoulos P, Parissis JT, Pieske B, Riley JP, Rosano GMC, Ruilope LM, Ruschitzka F, Rutten FH, van der Meer P; ESC Scientific Document Group. 2016 ESC Guidelines for the diagnosis and treatment of acute and chronic heart failure: The Task Force for the diagnosis and treatment of acute and chronic heart failure of the European Society of Cardiology (ESC) Developed with the special contribution of the Heart Failure Association (HFA) of the ESC. Eur Heart J, 2016; 37: 2129-2200

Ponikowski PP, Chua TP, Francis DP, Capucci A, Coats AJ, Piepoli MF. Muscle ergoreceptor overactivity reflects detorioration in clinical status and cadiorespratory reflex control in chronic heart failure. Circulation, 2001; 104: 2324-2330

Sbruzzi G, Ribeiro RA, Schaan BD, Signori LU, Silva AM, Irigoyen MC, Plentz RD. Functional electrical stimulation in the treatment of patients with chronic heart failure: a meta-analysis of randomized controlled trials. Eur J Cardiovas Prev Rehabil, 2010; 17: 254-260

Smart NA, Dieberg G, Giallauria F. Functional electrical stimulation for chronic heart failure: a metaanalysis. Int J Cardiol, 2012; doi:10.1016/j.icard.2011.12.019

Soska V, Dobsak P, Pohanka M, Spinarova L, Vitovec J, Krejci J, Hude P, Homolka P, Novakova M, Eicher JC, Wolf JE, Dusek L, Siegelova J. Exercise training combined with electrostimulation in rehabilitation 
of patients with chronic heart failure: a randomized trial. Biomed Pap Med Fac Univ Palacky Olomouc Czech Repub, 2014; 158(1): 98-106

Toy W, Burgess D, Wise K, Wise K, Nauman DJ, Crispell K, Hershberger RE. Comparative responsiveness of short form 12 and Minnesota Living with Heart Failure Questionnaire in patients with heart failure. J Card Fail, 2000; 6(2): 83-91

Van den Berg-Emons RJ, Bussmann JB, Balk AH, Stam HJ. Factors associated with the level of movementrelated everyday activity and quality of life in people with chronic heart failure. Phys Ther, 2005; 85: $1340-1348$

\section{Corresponding author:}

\section{Anna Polak}

Department of Physical Therapy, Academy of Physical Education, Mikolowska 72A str., 40-065 Katowice, Poland.

Fax number: 0048322075301

Phone number: 0048322075318

Email: a.polak@awf.katowice.pl 